\title{
Ion-induced damage and annealing of silicon. Molecular dynamics simulations*
}

\author{
David Humbird and David B. Graves ${ }^{\ddagger}$ \\ University of California at Berkeley, Berkeley, CA 94720, USA
}

\begin{abstract}
A study of the interactions of energetic argon ions with silicon surfaces using molecular dynamics simulations is reported. A dynamic balance between ion-induced damage and recrystallization of the surface is detected. By manipulating ion energy, argon ions are able to both create disordered regions near the surface, and recrystallize these disordered regions.
\end{abstract}

\section{INTRODUCTION}

Plasma processes are widely used to modify surfaces in many industries. A short list includes semiconductor fabrication, flat panel display manufacturing, manufacture of magnetic thin-film heads for information storage, microelectromechanical systems fabrication, thin-film deposition for decorative or functional coatings, polymer surface property modification to promote or inhibit adhesion, and so on.

This paper focuses on selected fundamental issues involved with the impact of energetic positive ions at surfaces exposed to low-pressure, nonequilibrium plasmas. As is well known, ion bombardment, and the creation of reactive free radicals in the plasma, are generally the major sources of near-surface modification. The objective is to learn more about basic questions of mass and energy transport, and the nature of these near-surface modifications from plasmas. The simulations described here are not intended to model a practical industrial plasma etch system.

\section{DESCRIPTION}

\section{General procedures}

Details of the simulation procedures can be obtained elsewhere [1-3], but a brief summary is included for completeness. All the simulations in this work used molecular dynamics (MD) with the velocity form of the Verlet algorithm [4]. The Stillinger-Weber potential [5] was used to calculate the forces between silicon atoms. A repulsive Molière pair potential [6] was used for all argon interactions. Periodic boundary conditions were imposed in the lateral dimensions to mimic an infinite system. The bottom two layers of all cells were held fixed to prevent bulk motion of the cell.

Events occurring in the relatively long times between impacts were not simulated, as it was assumed that nothing happens on the surface in this time, apart from cooling of the surface to room temperature, and desorption of any rare gas atoms. In standard impact simulation practice, the simulation cell is cooled after each impact by scaling the velocities of all particles. In all simulations reported here, the cell was returned to $300 \mathrm{~K}$ after each impact. The next impact then begins immediately. Since the length of clock-time represented by the simulation is thus undefined, ion quantities are reported in terms

\footnotetext{
*Lecture presented at the $15^{\text {th }}$ International Symposium on Plasma Chemistry, Orléans, France, 9-13 July 2001. Other presentations are presented in this issue, pp. 317-492.

${ }^{\ddagger}$ Corresponding author
} 
of fluence (in monolayers), rather than flux. One monolayer is defined to be a lateral plane of silicon atoms in the [100] direction, corresponding to about $7 \times 10^{14}$ atoms $\mathrm{cm}^{-2}$.

As a shorthand notation, we use the term "ion" to refer to energetic species in this paper. In fact, we assume that ions are neutralized just before impact and are therefore interacting with the surface atoms as neutrals. The graphics in this paper were created with VMD [7].

\section{RESULTS AND DISCUSSION}

\section{$\mathrm{Ar}^{+}$impacts on silicon}

The set of simulations reported here illustrate the effect of $\mathrm{Ar}^{+}$ions impacting silicon surfaces at normal incidence and a range of energies. After repeated impact, an initially crystalline Si surface will have its top layers rendered amorphous as shown in Fig. 1 for $100 \mathrm{eV}$ ions [8]. After about 0.5-0.8 ML fluence, the steady-state amorphous layer depth of about $9 \AA$ is reached. Amorphous layers were characterized by an order parameter relating the atoms' positions in the layer with their ideal crystalline positions. A cutoff value for this parameter, used to calculate the depth of the layer, was chosen by visual inspection. The interface between the crystalline and amorphous layers appears to act almost like a phase boundary. Animations made of the impacts show that atoms in the crystalline layer near the boundary will occasionally be disordered. However, several impacts later, this region will be recrystallized. The depth of the amorphous layer appears to be controlled by some dynamic balance between creation and destruction of defects and damage.

The thickness of the amorphous layer is a function of ion energy: the higher the energy, the deeper the amorphous layer. We postulated that if ions deposit more than some threshold amount of energy at some depth, the layer is rendered amorphous. However, it also suggests that a previously amorphized region near the boundary will be recrystallized if the deposited energy is not too high or too low. This proved to be the case. Figure 2 illustrates this process. On the left axis is plotted the thickness of the amorphous layer as a function of ion fluence. Snapshots of the side view of the layer are shown at different fluences. At zero fluence, the layer is crystalline. On the right axis is plotted the ion energy. From 0 to about 1.5 ML, $200 \mathrm{eV}$ ions impact the surface, and the amorphous layer depth approaches its steady-state value of about 15-20 ̊. From 1.5 ML to about 4.25 ML, the ion energy is reduced to $100 \mathrm{eV}$. The amorphous layer depth reduces to about 8-10 $\AA$ under $100 \mathrm{eV}$ ion bombardment. In other

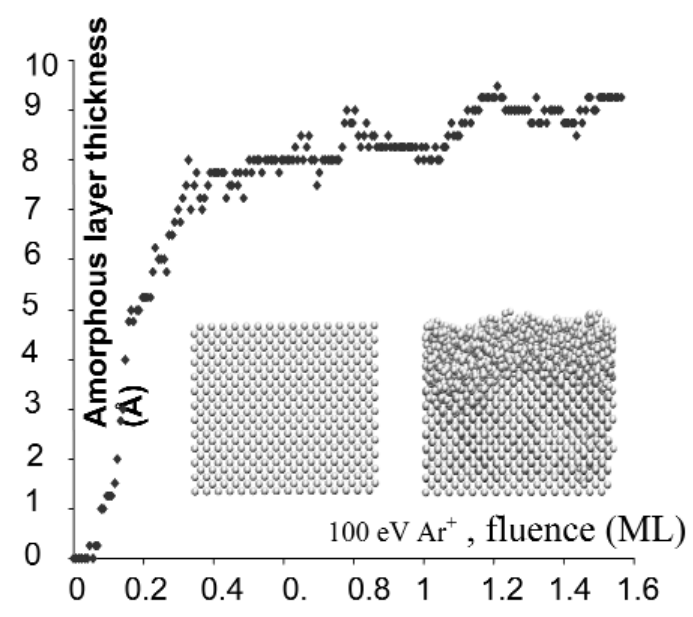

Fig. 1 Approach to steady-state of the amorphous layer thickness. Silicon surface under $100 \mathrm{eV} \mathrm{Ar}^{+}$ bombardment. Initial and final configurations are shown. 


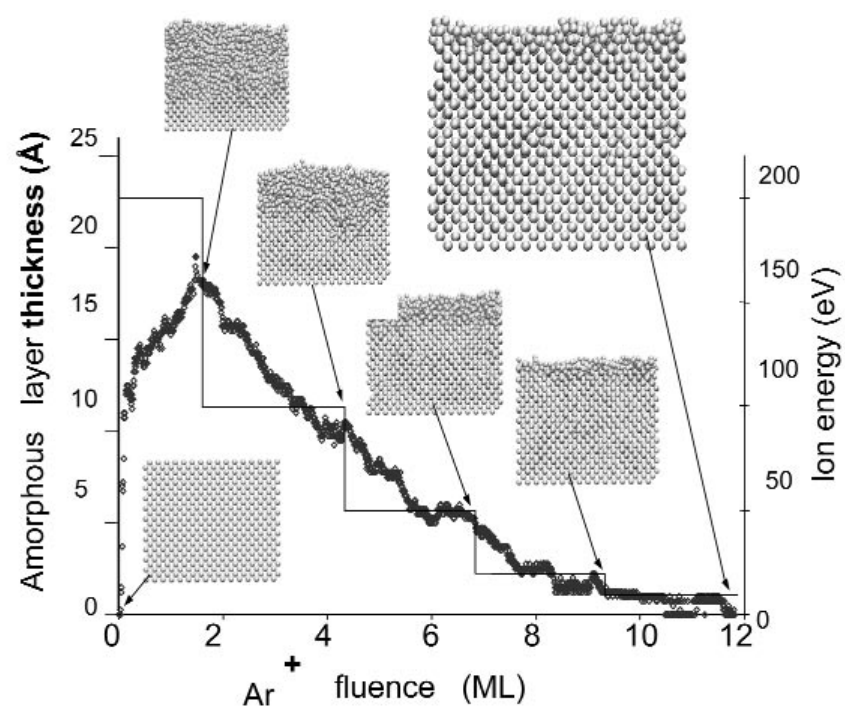

Fig. 2 Damage and subsequent annealing of silicon by $\mathrm{Ar}^{+}$ions. Snapshots of the cell are shown at various fluences. Final configuration enlarged to show defects.

words, formerly amorphous silicon is recrystallized when the ion energy is reduced. If this process is continued to $50 \mathrm{eV}$, then to $20 \mathrm{eV}$ and finally to $10 \mathrm{eV}$, the amorphous layer depth is reduced to only about 1-2 A. Essentially almost all but the top surface atoms are recrystallized by gradually lowering ion energy to $10 \mathrm{eV}$. A few defects can be seen in the layer, however, indicating that the ion-assisted annealing was not completely successful. In a related simulation, we found that by starting with $200 \mathrm{eV}$ ions and an amorphous layer depth of $15 \AA$, then immediately reducing the ion energy to $20 \mathrm{eV}$, the layer recrystallized much more slowly. Both simulations suggest that ions must deposit enough energy at the amorphous-crystalline boundary to allow atoms to rearrange in the epitaxial pattern. In the case of drastic energy reduction, only excursive ions have enough energy to penetrate to the boundary. This is known to have an application in ion beam-assisted silicon epitaxy: by firing an energetic Si into the deposited amorphous layer every so often, its final thickness is reduced, and annealing times are shortened $[9,10]$. Furthermore, there seems to be an optimal range of ion energy at the boundary, within which the layer will recrystallize. Too high an ion energy, and the disordered silicon atoms remain disordered, but if the energy is too low, the disordered atoms cannot move into the lower energy positions defined by the underlying crystalline atoms.

To help confirm this idea, we characterized the ion energy as a function of depth in the layer, and related this to the depth of the boundary. Figure 3 is a plot of the ion's remaining energy as a function of depth, for several ion energies, averaged over 1000 impacts each. The most energy is deposited at the surface of course, with the fraction remaining decreasing steadily with depth. The higher the initial energy, the greater the total depth of ion energy deposition. However, the key result is that the amorphous-crystalline boundary is very near the same location for each case-the depth at which the ion has about 10-12 eV remaining. A comparison between Figs. 2 and 3 shows the close correspondence between the depth at which the ion has about $10-12 \mathrm{eV}$ remaining and the depth of the amorphous-crystalline boundary for that energy. It appears that $10-12 \mathrm{eV}$ is the upper limit of ion energy at which the crystalline layer can survive. We note that this is consistent with the observation that $5-10 \mathrm{eV}$ ions appear not to damage a crystalline Si layer. 


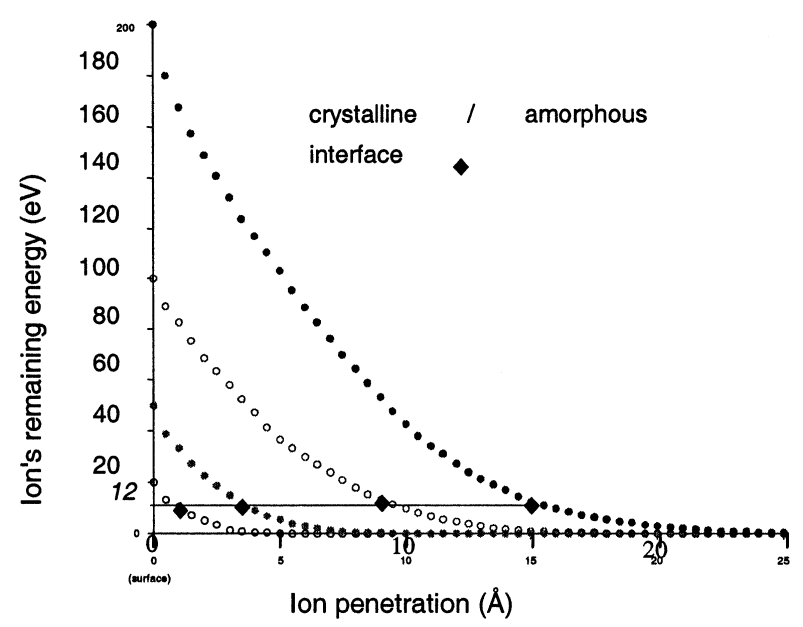

Fig. 3 Remaining energy profiles for argon ions in silicon at 20, 50, 100, and $200 \mathrm{eV}$. The depth of the amorphous-crystalline interface is indicated for each energy.

\section{CONCLUDING REMARKS}

The results presented here can be summarized as follows. Bombardment of silicon surfaces with $\mathrm{Ar}^{+}$ creates a near-surface amorphous layer. The depth of this layer depends on the ion energy, ranging from $1-2 \AA$ at $10 \mathrm{eV}$ to $15 \AA$ at $200 \mathrm{eV}$. It is possible to reverse the damage by gradually decreasing the ion energy, such that the ion energy remaining at the amorphous-crystalline interface is less than about $10-12 \mathrm{eV}$ and more than about $0.3-0.9 \mathrm{eV}$. A previously damaged silicon surface can be nearly perfectly (apart from a few defects deeper in the lattice) recrystallized up to the top $1-2 \AA$ at the surface. The simulations reported here and elsewhere suggest that it is possible to greatly extend the control of surface and near-surface properties, in part by utilizing atomistic simulations.

\section{REFERENCES}

1. M. E. Barone. Ph.D. thesis, U.C. Berkeley (1995).

2. B. A. Helmer. Ph.D. thesis, U.C. Berkeley (1998).

3. C. F. Abrams. Ph.D. thesis, U.C. Berkeley (2000).

4. W. C. Swope, H. C. Andersen, P. H. Berens, K. R. Wilson. J. Chem. Phys. 76, 637-649 (1982).

5. F. H. Stillinger and T. A. Weber. Phys. Rev. B 31, 5262-5271 (1985).

6. Torrens. Interatomic Potentials, Academic Press, New York (1972).

7. W. Humphrey, A. Dalke, K. Schulten. J. Mol. Graphics 14, 33-38 (1996).

8. M. E. Barone and D. B. Graves. J. Appl. Phys. 77, 1263-1274 (1995).

9. B. Strickland and C. Roland. Phys. Rev. B 51, 5061-5064 (1995).

10. H. Hensel and H. M. Urbassek. Phys. Rev. B 58, 2050-2054 (1998). 